\title{
Inhalt des neunten Bandes.
}

\author{
E r s t e s H e f t.
}

1. Beschreibung der beim Bau des Hafens am nenen Salzmagazine zu Berlin gebrauchten Wasserhebungs - Maschine. Von dem Herrn Bau-Conducteur Rosenbaum zu Berlin. . . . . . . . . . . . . . . Seile 1

2. Beschreibung der Irren-Zimmer und des Wasch - und Badehauses in der Irren-Heilanstalt zu Leubus in Schlesien. Von dem Königl. Bau-Inspector Hertn Rimann zu Wohlau. . . . . . . . . . . - 8

3. Nachrichten von der Belgischen Eisenbabn. (Fortsetzung von No. 9. und 12. Band 8.) . . . . . . . . . . . . . . . . . 33

4. Über die Kettenbrücke zu Freiburg in der Schweiz. Von einem Ungenannten. . . . . . . . . . . . . . . . -49

5. Andeutungen zar Charakteristik der Baustyle der Vorzeit; mit Hinweisung auf die bekannten Bauwerke und auf die Hülfsquellen zur Kenntnifs derselben. Von dem Königl. Regierangs - Bau-Inspector und Ingevieur-Premier-Lieutenant a. D. Herrn Emmich zu Frankfurt a. O. - - 53

6. Versuche über die Widerstandsfähigkeit der bekanntesten und nützlichsten Bausteine, welche das Rheinische Schiefergebirge und das daran grenzende Flötzgebirge an der Mosel und in den Ardennen liefern, angestellt in Festungs-Bauhofe zu Coblenz. Von dein Königl. Preuls. Ingenieur - Premier - Lieulenant Herrn Beise zu Coblenz. . . . . . -89

\section{Z w e i t es Heft.}

7. Vorlesungen über Eisenbahnen. Gehalten in der Ecole des ponts et chaussées zu Paris in den Jahren 1833 und 1834 von Herrn Minard. - 101

8. Bücher-Anzeige. . . . . . . . . . . . . . . -201

\section{D r i t t e s $H$ e f t.}

9. Vorschlag zu einer Gebäude-Gründung in besonders ungünstigem Boden. Von einem Ungenannten. . . . . . . . . . . -203 
10. Nachtrag zu der im 2ten Heft 8ten Bandes pag. 137 enthaltenen Flora ron Schlesien für das Bauwesen. Von dean Königl. Bau-Inspector Herrn Rimann zu Wohlau in Schlesien. . . . . . . . . . . . Seite 210

11. Einiges allgemein Verständliche über Eisenbahnen, insbesondere als Privat-Unternebmungen; für alle Diejenigen, welche sich dafür interessiren, und besonders fitr Diejenigen, so als Actionnairs daran Theil zu nelmen geneigt sind. Vom Herausgeber. . . . . . . . . . . -227

12. Fortsetzung der Abhandlung No.6. im 1. Hefte dieses Bandes. . . - -288

\section{Y i e r t e 8 H e $f$.}

13. Die neuen Kochófen in den Garnison-Lazarethen zu Coblenz, Luxemburg und Mainz. Yon dem Königl. Preufs. Ingenieur-PremierLieutenant Herrn Beise zu Coblenz. . . . . . . . . . . . . 299

14. Fortsețzung der Abpandlung von No. 6. Heft 1, und No. 12. Heft 3. dieses Bandes. . . . . . . . . . . . . . . . . . . . . -321

15. Ergebnisse einiger Untersuchungen über Kalk und Hörtel. Von Herrn Courtois, Brücken- und Wege-Ingenieur. (Aus den Annales des ponts et chaussées. 1834.) . . . . . . . . . . . . . . . . . . - 347

16. Fortsetzung der in 3, und 4. Hefte 8. Bandes und in 1. Hefte 9. Bandes dieses Journals enthaltenen Nachrichten von der Belgischen Eisenbahn, - 381

Einige Berichtigungen in diesem Bande. Im ersten Hefto.

Seite 92 lese man Laachener See ot. Lamser See.

- 96 Taf. 1. Spalte 2. Ranheil st. Reinheit.

- 99 Spaite 2. oben st. über.

Im dritten Hefle.

- 210 bis 226 lese mna überall $\mathrm{F}$ a milic stat $\mathrm{F}$ a mielie.

- 211 Acotyledoneen, Monocotyledoneen, Dicotyledoueen st. Acotylidoneo, Monocotylidoneu Dycotylidonen.

- 212 Z. 15 จ. o. parallela st. paralella.

- 212 - 23 v. o. Sareomycetes st. Succomiles,

- 213 - 4 v. o. Xylophagus st. Xilophagus.

- 215 und 217 mephiliseh st. mephytisch.

- 219 Z. 3 v. o. Hepaticae st. Stepaticae.

- 220 - 3 v. 0 . antipyretica st. antipretica.

$-223-13$ r. u. larynx st. laryx.

-291 §. 2. weich st. reich. 